\title{
LUTS in MTF Sex Reassignment Surgery: Is It Possible to Evaluate the Outcome?
}

\section{Carlo Melloni*}

Center for Genital Reconstructive and Aesthetic Surgery, Palermo, Italy

\begin{abstract}
Lower urinary tract symptoms (LUTS) refer to a group of medical symptoms, that affect approximately $40 \%$ of old men. It is a term used to describe a range of symptoms related to problems of the lower urinary tract (bladder, prostate and urethra).
\end{abstract}

Although it is known that in MTF transsexuals, LUTS may be common problems, little research effort has been devoted to use the correct instruments fot symptoms evaluation.

Is it possible to evaluate the surgical outcome and the presence of LUTS by using the existing questionnaieres?

\section{Introduction}

The equalization process in Male to Female (MTF) sex reassignment involves the correction of the external genitalia, breast enlargement, changes in body hair characteristics, and the redistribution of fat tissue in a female mode.

The surgical techniques used in male to female sex reassignment have as the principal goal (in addition to breast and other minor plastic reconfiguration) the creation of aesthetic and functional external female genitalia, with a normal-looking vestibulum, and a profound and compliant vaginal cavity. A variety of surgical options exists for vaginal reconstruction. Bizic et al. [1] described and reviewed several different reconstructive approaches and, in all techniques, the prostate remains untouched.

Lower urinary tract symptoms (LUTS) refer to a group of medical symptoms, that affect approximately $40 \%$ of old men [2]. It is a term used to describe a range of symptoms related to problems of the lower urinary tract (bladder, prostate and urethra). LUTS are broadly grouped into voiding (obstructive) symptoms or storage (irritative) symptoms. A man may have mainly voiding symptoms, mainly storage symptoms, or a combination of both $[3,4]$.

\section{Voiding or obstructive symptoms}

- Hesitancy - a longer than usual wait for the stream of urine to begin.

- Weak and poorly directed stream of urine.

- $\quad$ Straining to urinate.

- Dribbling after urination has finished or an irregular stream.

- Chronic urinary retention - not all the urine is passed from the bladder causing a need to urinate more often.

- Overflow or paradoxical incontinence - urine overflows from a full bladder uncontrollably even though normal urination can be difficult to start.

\section{Storage or irritative symptoms}

These are also symptoms typical of $\mathrm{OAB}$ (overactive bladder)

- Urgency - an urgent feeling of needing to urinate.

- $\quad$ Frequency - a short time between needing to urinate.
- Nocturia - a need to pass urine two or more times during the night.

- Urge incontinence - a sudden, intense urge to urinate followed by an uncontrolled loss of urine.

Quality of life is concerned with how a person perceives aspects of their life and how any illness impacts on aspects of their life that are important to them. LUTS can significantly reduce patients' quality of life and may point to serious pathology of the urogenital tract $[5,6]$.

Investigations about the impact of sex reassignment surgery on the lower urinary tract have so far concentrated on direct postoperative urological complications. Although urethral stenosis, infections, spraying of the urinary stream and incontinence have been reported immediately post-operatively [7-9], little research effort has been devoted to studying LUTS in this patient cohort, manly young men.

Aim of the study was to find out if it is possible to find instruments to evaluate if transsexuals have micturition disorders and - if so which, how satisfied they are with the current situation and how the prostate reacts to a life long substitution with cross gender hormones.

\section{Materials and Methods}

Urinary symptoms may affect Quality of Life (QoL). The level of distress depends on the frequency, urgency, involuntary leakage, the amount of leakage, and on the subjective experience of these symptoms. For example, fear of leakage or of the associated odor might lead to social withdrawal.

Views might differ between patients and clinicians. Thus, it is important to include reports of QoL and symptom severity directly from the patient. These reports must be collected in a standardized manner with validated self-assessment questionnaires. The literature

${ }^{*}$ Corresponding author: Carlo Melloni, Head of Gender Team, Center for Genita Reconstructive and Aesthetic Surgery, Palermo, Italy, Tel: 091528976; E-mail: studiomedico.melloni@gmail.com

Received: May 13, 2017; Accepted: June 30, 2017; Published: July 05, 2017

Citation: Melloni C (2017) LUTS in MTF Sex Reassignment Surgery: Is It Possible to Evaluate the Outcome? Reconstructive Surgery and Anaplastology 6: 168. doi: 10.4172/2161-1173.1000168

Copyright: (C) 2017 Melloni C. This is an open-access article istributed under the terms of the Creative Commons Attribution License, which permits unrestricted use, distribution, and reproduction in any medium, provided the original author and source are credited. 
review search strategy utilized PubMed web search engine and Medline database. The following keywords were used in various combinations: LUTS, Gender Dysphoria, Transgender, Transsexuals, Quality of Life, Sexual Quality of Life, Urinary infection, Micturition. There were no date restrictions, but only English language articles were used. After a deep search, we decided to use, for LUTS evaluation, these well established and validate questionnaires:

\section{IPSS (International Prostatic Symptoms Score).}

ICIQ-LUTSqol (International Consultation on Incontinence Modular Questionnaire on LUTS and Quality of Life).

\section{OABq-SF (Overactive Bladder Questionnaire-Short Form).}

We decideded to use IPSS tool because of the persistency of the prostate in MtF procedure. The shortening of urethra and the modification of the position in a feminine way, lead us to use the ICIQLUTSqol and OABq-SF.

\section{Discussion}

There's a plethora of studies and questionnaires commonly used to evaluate urinary symptoms and their impact on patient quality of life. Among that huge number of tools we've selected three questionnaires that we've considered useful for our study.

\section{IPSS (International Prostate Symptoms Score)}

The International Prostate Symptom Score (IPSS) [10] is an 8 questions (7 symptom questions +1 quality of life question) written screening tool used to screen for, rapidly diagnose, track the symptoms of, and suggest management of the symptoms of the disease benign prostatic hyperplasia (BPH). The 7 symptoms questions include feeling of incomplete bladder emptying, frequency, intermittency, urgency, weak stream, straining and nocturia, each referring to during the last month (4 weeks).

The IPSS was designed to be self-administered by the patient, with speed and ease in mind. Hence, it can be used in both urology clinics as well as the clinics of primary care physicians (i.e., by general practitioners) for the diagnosis of $\mathrm{BPH}$. Additionally, the IPSS can be performed multiple times to compare the progression of symptoms and their severity over months and years.

In addition to diagnosis and charting disease progression, the IPSS is effective in helping to determine treatment for patients.

Scoring: Each question consider a score from 1 to 5 for a total of maximum 35 points. The 8 th question of quality of life is assigned a score of 1 to 6 .

The first seven questions of the I-PSS are identical to the questions appearing on the American Urological Association (AUA) Symptom Index which currently categorizes symptoms as follows:

- Mild (symptom score less than of equal to 7)

- Moderate (symptom score range 8-19)

- Severe (symptom score range 20-35)

\section{ICIQ-LUTSqol}

The ICIQ-LUTSqol [9] (International Consultation on Incontinence Modular Questionnaire on LUTS and Quality of Life) is a psychometrically patient-completed questionnaire evaluating quality of life $(\mathrm{Q} o \mathrm{~L})$ in urinary incontinent patients for use in research and clinical practice across the world. The ICIQ-LUTSqol provides a detailed and robust measure to assess the impact of urinary incontinence on quality of life with particular reference to social effects. It is an ideal research tool as it explores in detail the impact on patients' lives of urinary incontinence. The Second International Consultation on Incontinence recommended that all randomized trials evaluating treatments for incontinence should employ standardized and validated questionnaires to assess their impact on patient outcome. The ICIQ-LUTSqol provides a robust measure for this purpose.

Scoring: The condition-specific QoL questionnaire (ICIQLUTSqol) consists of 19 items concerning different aspects of everyday life that might be affected by leakage or other bladder conditions.

Part A of each question is entered into the outcomes calculator and contributes to the overall score.

Part B of each question is a Visual Analogue Scale (VAS) bother score from 0 -10 which does not contribute to the overall score but is intended as a guide to clinicians to indicate the overall bother experienced by the patient. The VAS bother scores, including question 22 , are not entered into the outcomes calculator.

Each item provided four choices, which were rated 1="not at all/ never", 2="slightly/sometimes", 3="moderately/often", or 4="a lot/very much/all the time". The points are summed to an overall score ranging from 19 to 76 points. A higher score indicates a larger impact on quality of life. Three of the items concern personal relationships, and have an additional response: "not applicable." The additional response was considered equivalent to no effect on this aspect of quality of life (score $=1$ )

\section{OAB-q SF}

The Overactive Bladder Questionnaire (OAB-q) was developed to assess symptom bother and the impact of overactive bladder $(\mathrm{OAB})$ on health-related quality of life (HRQL). The instrument was developed and validated in both continent and incontinent $\mathrm{OAB}$ patients, including both men and women [11].

A short form of the original OAB-q was derived to provide a "quick" assessment of symptom bother and global HRQL. This "short form", called the OAB-q SF, consists of a 6-item symptom bother scale and a 13-item HRQL scale. The OAB-q SF should be used when the assessment of symptom bother is desired, but when a global assessment of HRQL, rather than specific domains of HRQL, is desired. The OAB-q SF uses a 4 week recall period [12-15].

Scoring: Scores have a possible range of $0-100$. Higher scores on the symptom bother scale indicate greater symptom severity/bother or impact.

\section{Conclusion}

The development of LUTS is a common problem after surgery, and patients who consider sex reassignment should be informed about these side effects preoperatively.

Reporting on surgical outcomes after SRS is challenging. Sutcliffe et al. performed a representative systematic review on main literature databases showing important limitations regarding surgical outcomes and treatment options. Indeed, conducting a long term prospective study with this heterogeneous group of patients is not easy. The main barrier on describing surgical outcomes is the lack of comparable publications detailing the techniques used and their complications, not to mention on how to manage them, precluding reproduction of the available surgical methods. 
Eldh et al. and Lawrence concluded that surgical outcomes have a positive impact on patient's outcome and satisfaction.

It is clear that using 3 complete questionnaires in the same time may be boring for patients and response may be influenced by this.

Next step of our study will be the creation of a new questionnaire, derived from others, to conduct a prospective study on a cohort of MTF patients.

\section{References}

1. Bizic M, Kojovic V, Duisin D, Stanojevic D, Vujovic S, et al. (2014) An overview of neovaginal reconstruction options in male to female transsexuals. Scientific World Journal 2014: 638919.

2. Abrams $P$ (1994) New words for old: Lower urinary tract symptoms for "prostatism". BMJ 308: 929-930.

3. Welch G, Kawachi I, Barry MJ, Giovannucci E, Colditz GA, et al. (1998) Distinction between symptoms of voiding and filling in benign prostatic hyperplasia: Findings from the health professionals follow-up study. Urology 51: $422-427$

4. Roehrborn CG, McConnell JD, Saltzman B (2002) Storage (irritative) and voiding (obstructive) symptoms as predictors of benign prostatic hyperplasia progression and related outcomes. Eur Urol 42: 1-6.

5. Welch G, Weinger K, Barry MJ (2002) Quality-of-life impact of lower urinary tract symptom severity: Results from the health professionals follow-up study. Urology 59: 245-250.
6. Barry MJ (2001) Evaluation of symptoms and quality of life in men with benign prostatic hyperplasia. Urology 58: 25-32.

7. Fitzpatrick C, Swierzewski SJ, McGuire EJ (1993) Periurethral collagen for urinary incontinence after gender reassignment surgery. Urology 42: 458-460.

8. Noort V, Dirk E, Nicolai JP (1993) Comparison of two methods of vagina construction in transsexuals. Plast Reconstr Surg 91: 1308-1315.

9. Cavadas PC, Landin L (2005) Treatment of urethral stricture in a femaleto-male transsexual with a tubulized flap from the labia minora. J Reconstr Microsurg 21: 153-156.

10. Plante M, Corcos J, Gregoire I, Belanger MF, Brock G, et al. (1996) The international prostate symptom score: physician versus self-administration in the quantification of symptomatology. Urology 47: 326-328.

11. Coyne K, Revicki D, Hunt T, Corey R, Stewart W, et al. (2002) Psychometric validation of an overactive bladder symptom and health-related quality of life questionnaire: The OAB-q. Qual Life Res 11: 563-574.

12. Sutcliffe PA (2009) Evaluation of surgical procedures for sex reassignment: a systematic review. J Plast Reconstr Aesthet Surg 62: 294-306.

13. Eldh J, Berg A, Gustafsson M (1997) Long-term follow up after sex reassignment surgery. Scand J Plast Reconstr Surg Hand Surg 31: 39-45.

14. Lawrence AA (2003) Factors associated with satisfaction or regret following male-to-female sex reassignment surgery. Arch Sex Behav 32: 299-315.

15. Avery K, Donovan J, Peters TJ, Shaw C, Gotoh M, et al. (2004) ICIQ: A brief and robust measure for evaluating the symptoms and impact of urinary incontinence. Neurourol Urodyn 23: 322-330. 